\title{
Laparoscopic-Endoscopic Rendezvous Resection of Upper Gastrointestinal Tumors
}

\author{
D. Schubert ${ }^{\mathrm{a}}$ \\ R. Kuhn ${ }^{a}$ \\ G. Nestler ${ }^{a}$ \\ S. Kahl ${ }^{b}$ \\ M.P. Ebert ${ }^{\text {b }}$ \\ P. Malfertheiner ${ }^{b}$ \\ H. Lippert ${ }^{a}$ M. Pross ${ }^{a}$ \\ ${ }^{a}$ Department of General, Visceral and Vascular Surgery, and ${ }^{b}$ Department of Gastroenterology, Hepatology and \\ Infectious Diseases, Faculty of Medicine, Otto von Guericke University Magdeburg, Magdeburg, Germany
}

\section{Key Words}

Gastric tumor · Laparoendoscopic surgery · Early gastric cancer - Gastrointestinal stromal tumor • Rendezvous resection · Surgery

\begin{abstract}
Background: Endoscopic and laparoscopic local resection of gastric tumors has increasingly been performed in recent years. This article describes the technical considerations and early results of laparoscopic-endoscopic rendezvous resection of gastric lesions. Patients and Methods: Rendezvous resection was performed in 26 patients with submucosal gastric tumors $(n=22)$ and early gastric cancer $(n=4)$. Laparoscopic wedge resection (LWR) was performed in 16 patients with anterior wall tumors and laparoscopic intragastric resection (LIR) in 7 patients with posterior wall tumors. Conversion to open surgery was carried out in 3 cases. Results: Operation times were $53 \mathrm{~min}$ (range 35-115) for LWR and $83 \mathrm{~min}$ (range 56-130) for LIR, respectively. In submucosal lesions the mean tumor size was $36 \mathrm{~mm}$ (range 16-47) and in early gastric cancer $17.3 \mathrm{~mm}$ (range 16-20). Rendezvous resection was performed with curative intent and clear resection margins in all patients without lymphatic or vessel permeation. Postoperative complications occurred in 2 patients. After a mean follow-up of 22.8
\end{abstract}

months (range 2-71), no local recurrence or metastatic disease and no tumor-related death were observed. Conclusions: When selected properly, the laparoscopic-endoscopic approach is considered to be curative and safe for resection of localized gastric tumors.

Copyright (c) 2005 S. Karger AG, Basel

\section{Introduction}

Since the introduction of laparoscopy in 1910 [1], technological advances and research documenting the benefits of minimally invasive surgery have expanded its applicability to many general surgery procedures, including the treatment of gastric disease. Although the literature is currently limited to small series and case reports of this technique, it is apparent that the laparoscopic treatment of a wide variety of gastric pathologies, including benign neoplasms, premalignant lesions, tumors with unclear histology and early gastric cancer, is a viable option [2-6]. The localization, size and depth of wall invasion, and histological type of the tumor are essential criteria for the selection of a laparoscopic approach. At present, only localized gastric lesions should be considered for minimally invasive treatment. Careful preoperative planning and patient selection are crucial for the success of laparoscopic-endoscopic gastric rendezvous resection as well as for

\section{KARGER}

Fax +4161306 1234 E-Mail karger@karger.ch www.karger.com
Daniel Schubert, MD

Klinik für Allgemein-, Viszeral- und Gefässchirurgie

Otto-von-Guericke Universität Magdeburg

Leipziger Strasse 44, DE-39120 Magdeburg (Germany)

Tel. +49 3916715500, Fax +49 3916715570 , E-Mail schubertdr@hotmail.com 
optimal local and oncological outcome. Preoperative staging must be precise. Therefore, patients with early gastric cancer with limited gastric wall invasion (T1m) and without the presence of suspect lymph nodes are potential candidates for minimally invasive local treatment [7]. Under these conditions, laparoscopic-endoscopic rendezvous resection is an alternative surgical procedure to endoscopic mucosal resection for the local treatment of such tumors with curative intent. Submucosal tumors of the stomach display a wide spectrum of histopathologic and prognostic characteristics. Biopsies obtained using endoscopic techniques often do not provide the representative histologic sample needed for further therapeutic decisions. Full-thickness resection with a margin of healthy tissue around the tumor is recommended to ensure complete clearance of the neoplastic tissue. In this situation, laparoscopic-endoscopic rendezvous resection of a submucosal gastric tumor serves firstly as a diagnostic tool which allows complete histological examination of the resected specimen and provides the basis for further therapeutic decisions [8-10]. This article describes the technical considerations and early results of laparoscopic-endoscopic rendezvous resection of gastric lesions.

\section{Diagnostic Evaluation}

Careful preoperative planning, patient selection and diagnostic evaluation are crucial for the success of laparoscopic-endoscopic rendezvous resection as well as for optimal local and oncological outcome.

In the majority of cases, upper endoscopy is the first diagnostic step to evaluate a gastric lesion. Endoscopic biopsies are imperative in identifying a gastric carcinoma, however, the utility of this technique in regard to submucosal stromal tumors is limited. Endoscopic ultrasound (EUS) is a valuable technique in the diagnosis and staging of gastric cancer in experienced hands $[11,12]$. It has also been used effectively to identify gastric submucosal tumors. The effectiveness of EUS has been demonstrated to be at best for leiomyomas and poor for other submucosal tumors [13]. However, the level of experience of the endosonographer is a critical factor, as greater experience is associated with a high degree of accuracy [13]. EUS is imperative prior to laparoscopic gastric resection to objectivate the depth of wall invasion, and tumor expansion inside the gastric wall and to evaluate the local and regional lymph nodes. Fluoroscopic studies may augment endoscopy by delineating a submucosal tumor.
Abdominal sonography and computed tomography serve as a useful adjunct in the identification of metastatic disease and other pathologies of the digestive tract, although endosonography may be the superior modality in identifying local and regional disease [14]. Finally, a chest X-ray should complement the diagnostic evaluation of a gastric lesion.

\section{Technique of Laparoscopic-Endoscopic Gastric Rendezvous Resection}

Laparoscopic-endoscopic rendezvous resection of upper gastrointestinal tumors is performed under general anesthesia. The patient is generally placed in a supine or in a mild reverse Trendelenburg position. The monitors as well as the endoscopist are positioned near the head of the patient. The surgeon stands between the patient's legs and is thus aligned with the stomach and the monitor. The peritoneal cavity may be accessed via the closed Veress needle technique or the open method according to the surgeon's preference. The camera port is placed in the midline approximately one-third of the distance from the umbilicus to the xiphoid process. The remaining 2-4 trocars are placed in the right or left upper or middle quadrant depending on tumor size and localization. An attempt should be made to maintain a distance of $8-10 \mathrm{~cm}$ between the trocar sites to ensure optimal handling. The basic instrumentarium for rendezvous gastric resection includes atraumatic graspers, an endoscopic stapling device, electrocautery or an ultrasonic dissector and an extraction bag for the specimen. The simultaneous performance of laparoscopy and upper endoscopy is a prerequisite for this approach. The exact position of the tumor is determined by laparoscopy and endoscopic viewing when laparoscopically manipulating along the gastric wall. Depending on the location and size of the tumor, different strategies are described to approach a gastric lesion.

\section{Laparoscopic Wedge Resection (Tumors of the Anterior Gastric Wall)}

The development of articulating endoscopic stapling devices has facilitated the wedge excision of gastric tumors of the anterior wall, lesser and greater curvature and fundus with adequate distance to the cardia or pylorus, up to a size of approximately $5 \mathrm{~cm}$. The gastric wall tumor may be lifted with stay sutures which were initially placed 


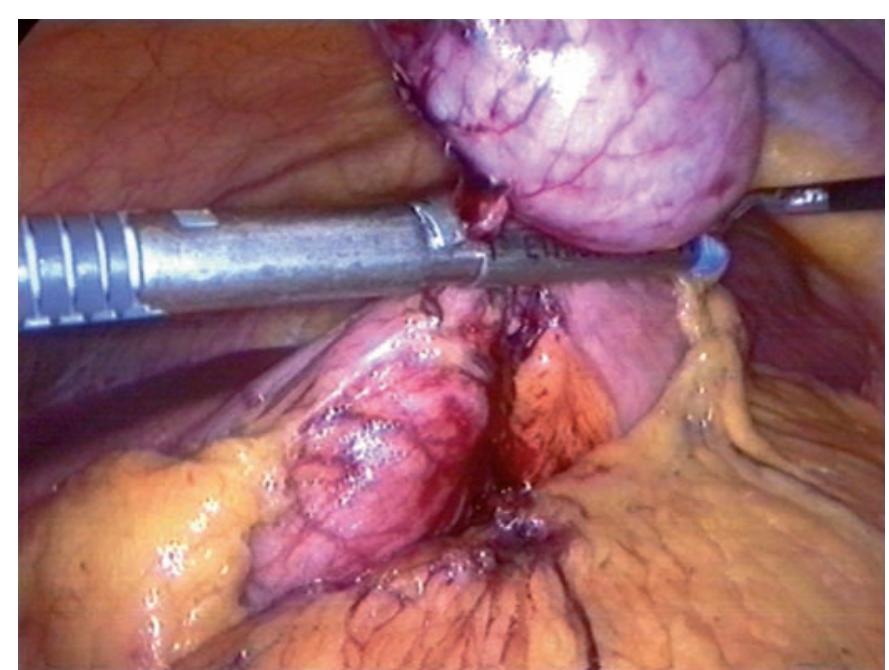

Fig. 1. Laparascopic wedge resection using an endoscopic stapling device after lifting a gastric wall tumor.

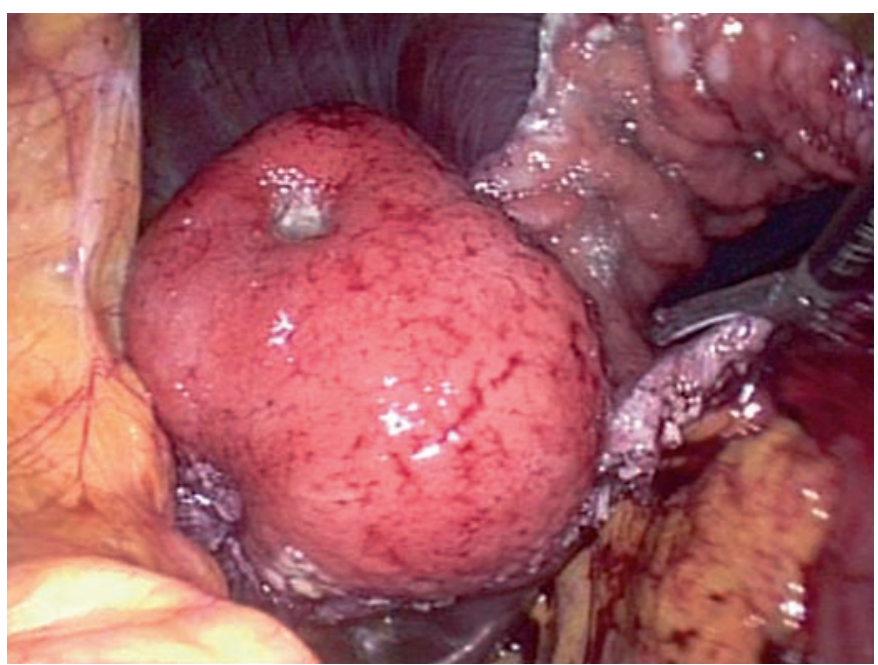

Fig. 2. Laparoscopic excision of a large gastrointestinal stromal tumor of the anterior wall using Ultracision ${ }^{\circledR}$; opened stomach is visible (right part of the picture).

through the normal gastric wall near the tumor, or with a laparoscopic Babcock clamp and then excised by firing a stapler across the tumor-free base of the lesion (fig. 1). This approach may require sequential application of the stapling device until the lesion is fully excised. If the tumor is located along the lesser or greater curvature, the omentum minus or majus can be divided from the stomach with clips or with ultrasonic dissection. This tech-

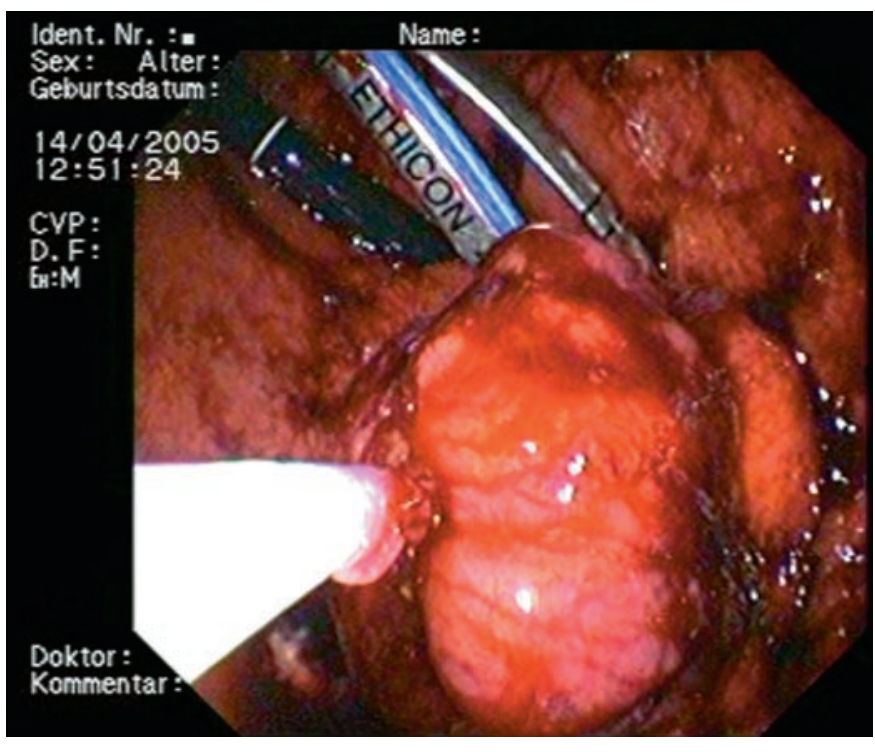

Fig. 3. A large gastrointestinal stromal tumor is grasped with a polypectomy snare, lifted into the stomach and excised intragastrically using an endostapler; endoscope in inversion is visible at 11 o'clock.

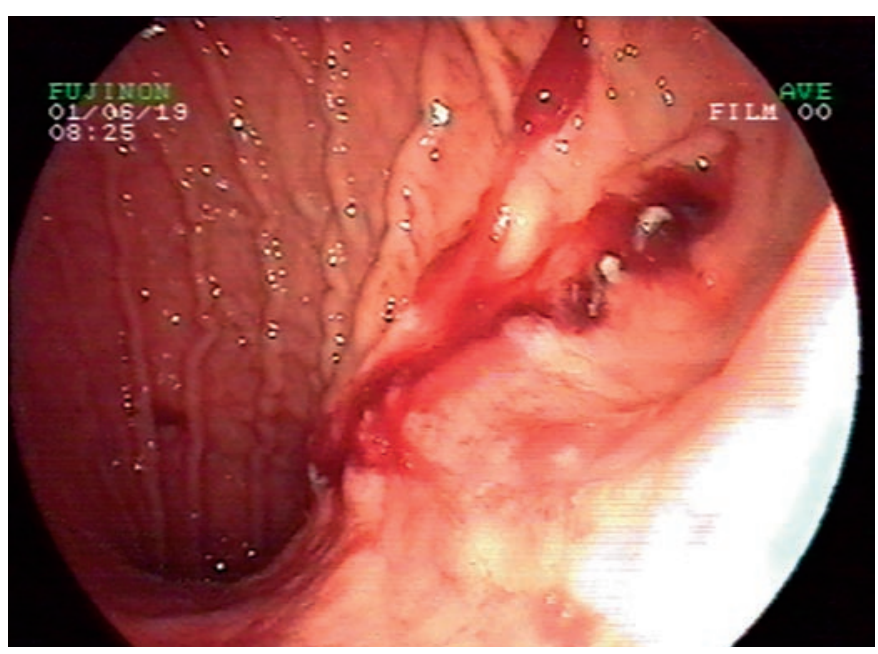

Fig. 4. Endoscopic view of a bloodless and tight stapler line after laparoscopic wedge resection.

nique should be employed under endoscopic guidance and with careful consideration of the resultant configuration of the stomach after resection to avoid gastric outlet obstruction. Exophytic stromal tumors are quite suitable for wedge resection with endoscopic stapling devices, whereas endophytic or large tumors may be difficult to resect with this approach due to the degree of stomach wall involvement. If the gastric lumen will be severely 
compromised by this technique, excision of the lesion using laparoscopic scissors and electrocautery or an ultrasonic dissector (fig. 2), followed by intracorporeal suture closure, may produce a more satisfactory result [15].

Additionally, laparoscopic extramucosal enucleation is an alternative technique for small benign tumors.

\section{Laparoscopic Intragastric Resection (Tumors of the Posterior Gastric Wall)}

Tumors of the posterior wall may be excised by a transgastric or intragastric approach. Again, intraoperative endoscopy aids in tumor localization. After explorative laparoscopy, the intra-abdominal gas pressure is reduced and an anterior gastrostomy overlying the tumor is made with electrocautery or ultrasonic dissection. It is helpful to place two stay sutures through the gastric wall, so that the anterior gastric wall can be lifted to the abdominal wall. A purse-string suture is then made between these two stay sutures. The gastrostomy can be carried out through the center of the purse-string suture. Afterwards, one up to three self-dilating trocars are introduced into the stomach under endoscopic guidance. It is important to choose the correct location for the gastrostomy (gastrostomies), so that the direction of introduction of the laparoscopic instruments used later provides the optimal route to the center of the tumor. We recommend that a purse-string suture should always be placed in order to achieve hermetic closure and hemostasis of the gastric wall during resection. Using the intragastric view provided by the video gastroscope, the tumor is firmly grasped intragastrically with a polypectomy snare or with intragastrically placed laparoscopic instruments and is then lifted into the stomach cavity. Thus, an endoscopic stapling device or an electrosurgical instrument is placed through an intragastric trocar into the stomach. The tumor is then excised with an endostapler (fig. 3) or electrosurgical (ultrasonic) dissection, placed in a retrieval bag and removed endoscopically or laparoscopically, according to the size of the tumor. The anterior gastrostomy is closed with an endoscopic stapling device or with intracorporeal running suture. Some authors recommend to place an additional laparoscopic camera intragastrically that provides for the intragastric view under these conditions $[16,17]$. Alternatively, division of the short gastric vessels facilitates exposure of more remote posterior tumors. Once these vessels are transected, the stomach can be axially rotated to provide extragastric access to posterior tumors along the lesser curvature and cardia.

\section{Intraoperative Flexible Endoscopy}

Endoscopy should always be performed either as part of the preoperative evaluation or in the intraoperative setting, where it has several advantages. The addition of flexible endoscopy enhances laparoscopic gastric resection, because decreased tactile feedback is inherent to the procedure [18]. Endoscopic translumination facilitates visualization of the gastric lesion from the laparoscopic view. Additionally, endoscopic visualization of the gastric pathology eliminates the need for preoperative tattooing of the lesion. Intraoperative endoscopy also provides a means to test the integrity of the gastric closure or anastomosis after resection (fig. 4). The stomach is insufflated under saline solution or filled with methylene blue to evaluate the stapled or sutured gastric closure for leaks. Although intraoperative endoscopy has clear advantages, the degree to endoscopic insufflation should be limited to avoid multiple loops of dilated small bowel and a subsequent decreased working space.

\section{Patients and Results}

Laparoscopic-endoscopic rendezvous resection was performed in 26 patients with gastric tumors between January 1999 and April 2005 in our department. These included 16 men and 10 women with a median age of 67.2 years (range 47-84). 22 patients had a submucosal gastric tumor and 4 had an early gastric cancer. In 24 cases the gastric tumor was diagnosed by primary upper endoscopy. In 2 patients a submucosal gastric tumor was detected by computed tomography which was carried out for other reasons. Preoperative histological clarification of a suspected early gastric cancer was successful by endoscopic biopsies in 3 cases. The tumor was restricted to the mucosa in all cases of an early carcinoma without the presence of suspect local or regional lymph nodes, assessed by EUS. In an 84-year-old woman, full-thickness resection in rendezvous technique was carried out after incomplete endoscopic mucosal resection of an early gastric cancer. Definite preoperative clarification of the tumor type was feasible only in 9 of 22 patients with submucosal gastric tumors, despite multiple biopsies and extensive diagnostics.

Laparoscopic wedge resection (LWR) was performed in 12 patients with a submucosal stromal tumor and in 4 patients with early gastric cancer of the anterior gastric wall. Laparoscopic intragastric resection (LIR) was carried out in 7 patients with submucosal stromal tumors of 
the posterior gastric wall. Conversion to open surgery was performed in 3 cases, once, because of prior abdominal surgery and in 2 cases due to the tumor size $(>5 \mathrm{~cm})$ and the location of the lesion near the cardia. Operation times were $53 \mathrm{~min}$ (range 35-115) for LWR and $83 \mathrm{~min}$ (range 56-130) for LIR, respectively. In submucosal lesions the mean tumor size was $36 \mathrm{~mm}$ (range 16-47) and in 3 cases of early gastric cancer 16, 17 and $20 \mathrm{~mm}$. Postoperative complications occurred in 2 patients. CT-guided percutaneous placement of a pigtail catheter 6 days after laparoscopic-endoscopic rendezvous resection was required in 1 case because of a subphrenic abscess formation. Another patient developed a port side hernia that needed further surgical treatment. No fatal outcome had to be registered. Patients were discharged after a median hospital stay of 5.6 days (range 2-12) after operation. Laparoscopic-endoscopic rendezvous resection was performed with curative intent and clear resection margins in all patients. Postoperative histologic diagnosis indicated an adenocarcinoma (well differentiated, without lymphatic or vessel permeation) confined to the mucosa in 3 patients with early gastric cancer. Isolated tumor cells in the submucosal layer were found in the full-thickness specimen after gastric rendezvous resection following incomplete endoscopic mucosal resection of an early gastric cancer in an 84-year-old woman. This patient allowed no further treatment because of her great age and considerable co-morbidity. Postoperative histologic examination of locally excised submucosal tumors detected ectopic pancreatic tissue in 1 case, lipomas in 2 cases, leiomyomas in 3 cases and gastric stromal tumors without malignant criteria in 16 cases. After a mean follow-up of 22.8 months (range 2-71) no local recurrence or metastatic disease and no tumor-related death were observed.

\section{Discussion}

Laparoscopic-endoscopic rendezvous resection of gastric lesions is both feasible and safe. Basically, only locally limited gastric lesions should be considered for minimally invasive local treatment at present. Locally advanced tumors, lymphatic and metastatic disease are contraindications $[5,6]$. Therefore, careful preoperative planning, patient selection and extensive diagnostics are crucial for the success of gastric rendezvous resection as well as for optimal oncological outcome.

For this minimally invasive approach to be successful, close cooperation between surgeon, endoscopist and pa- thologist is required. Important points that need to be discussed are: (1) localization of the tumor; (2) size of the tumor, (3) depth of gastric wall invasion and expansion of the tumor, and (4) histological type of the tumor.

In the majority of cases, upper endoscopy is the first diagnostic step to evaluate a gastric lesion. Endoscopic biopsies are imperative and usually successful in identifying a carcinoma in a gastric lesion, however, the utility of this technique with regard to submucosal stromal tumors is limited [19]. Biopsies of submucosal stromal tumors are often superficial to the lesion and nondiagnostic. Examination of several microscopic fields of the full pathologic specimen is required to determine the malignant potential of such tumors. In many cases, only full-thickness resection can classify the accurate histologic diagnosis of the tumor type. EUS is a valuable technique in the diagnosis and staging of gastric cancer in experienced hands $[11,12]$. At present, it is the most important diagnostic tool to evaluate the depth of wall invasion of a mucosal gastric lesion. The diagnostic accuracy to objectivate the depth of wall invasion has been demonstrated to be between 75 and 95\% [11, 12]. Accuracy of discrimination of $\mathrm{T} 1$ and $\mathrm{T} 2$ tumors ranges between 63 and $83 \%$, whereas mostly an overstaging is noted [12]. However, the level of experience of the endosonographer is a critical factor, as greater experience is associated with a high degree of accuracy [13]. EUS has also been used effectively to identify gastric submucosal tumors. Compared to malignant tumors, a benign stromal tumor is characterized by an anechogenic, homogenous appearance with regular margins [20]. The effectiveness of EUS has been demonstrated to be at best for leiomyomas and poor for other submucosal tumors [13]. EUS is imperative prior to laparoscopic gastric resection to objectivate the depth of wall invasion, and tumor expansion inside the gastric wall and to evaluate the local and regional lymph nodes.

Two major factors associated with the prognosis of patients with gastric cancer are the depth of gastric wall invasion and status of lymph node metastasis [21, 22]. The most important factor influencing the survival of patients with early gastric cancer is the status of lymph node metastasis [23, 24]. The incidence of lymph node metastasis in large series of early gastric cancer ranges from 1 to $3 \%$ for tumors confined to the mucosa [24-26] and from 11 to $20 \%$ for tumors invading the submucosa [2528]. Against this background, local endoscopic or laparoscopic resection of early gastric cancer restricted to the mucosa has increasingly been performed in recent years [29-36]. In a review, Adachi et al. [7] had searched for histological and morphological parameters which should 
serve as indicators for local resection of an early gastric cancer. Accordingly, local endoscopic treatment is adequate in patients with early gastric cancer confined to the mucosa, measuring $<2 \mathrm{~cm}$, grossly elevated or depressed type without ulceration and histologically well or moderately differentiated type [7]. When submucosal invasion, vessel permeation, or incomplete resection margins are recognized on the resected specimen, additional surgical treatment is recommended. Recently, especially in Japan, these criteria were expanded partially for tumors measuring $<3 \mathrm{~cm}$, poorly differentiated tumors and for tumors invading the upper submucosal layer [37].

In particular, endoscopic mucosal resection has been employed in patients with early gastric cancer in recent years. A local recurrence rate of $2-17 \%$ during long-term follow-up was noted after this procedure [29-32]. The number of $\mathrm{R} 0$ resections ranged between 62 and $85 \%$ in several large prospective series [29-32]. In recent years, various authors have reported promising results after laparoscopic-endoscopic rendezvous resection of $\mathrm{T} 1 \mathrm{~m}$ gastric carcinomas with local recurrence in $0-3.6 \%$ during a mean follow-up of 12-93 months and with $\mathrm{R} 0$ resection in all patients [33-36]. Consequently, it may be suggested that laparoscopic-endoscopic rendezvous resection provides at least the same oncological outcome as endoscopic mucosal resection in patients with early gastric cancer. Due to the comparatively small number of patients with early gastric cancer treated by laparoscopic-endoscopic rendezvous resection so far, these results require validation and prospective controlled randomized trials are needed. In our opinion, laparoscopic-endoscopic rendezvous resection should be considered as an alternative surgical approach to early gastric cancer and not as rivalry to endoscopic mucosal resection. Another advantage of that procedure, in addition to full-thickness resection of a gastric lesion, is the feasibility of local lymph node dissection [35]. However, the diagnostic and therapeutic benefits of local lymph node sampling in patients with early gastric cancer are not definitely confirmed up to now and the clinical implications and prognostic significance of lymph node dissection are controversial.

Submucosal gastric tumors, in particular benign lesion and gastric stromal tumors without malignant potential, are ideal candidates for laparoscopic-endoscopic rendezvous resection.

As the histology of such tumors is often preoperatively unknown, full-thickness resection with a margin of healthy tissue around the tumor is recommended to ensure complete clearance of the neoplastic tissue. Examination of several microscopic fields of the full pathologic specimen is required to determine the malignant potential of gastrointestinal stromal tumors (GISTs). In this situation, laparoscopic-endoscopic rendezvous resection of a submucosal tumor serves firstly as a diagnostic tool which allows complete histological examination of the resected specimen. Only the pathologist can answer the question if minimally invasive local treatment was performed with curative intent. Otherwise, subsequent radical organ resection is required. At present, surgical resection is the only way to treat GISTs with curative intent. Initially, these mesenchymal neoplasms are characterized to be slow and localized in growth [38]. Lymph node or distant metastases in patients with smaller GISTs are a rarity, which is why radical organ resection is usually not required [38, 39]. More recent data suggest that anatomic location is a prognostic factor with a trend for esophageal and gastric tumors to have the best prognosis, but the basis for these differences remains uncertain [38]. The morphologic features that have gained greatest acceptance as being predictive of outcome, behavior and metastatic risk in GISTs are mitotic rate and tumor size. Lesions measuring $<5 \mathrm{~cm}$ with a mitotic rate $<10$ per 50 high-power fields are labeled as potential benign or borderline tumors with a low or intermediate risk, respectively, whereas a tumor size $>5 \mathrm{~cm}$ and a mitotic count $>10$ per 50 high-power fields indicate malignant potential and aggressive behavior [38]. In consideration of these criteria, local minimal invasive treatment is currently adequate in GISTs measuring $<5 \mathrm{~cm}$ with a mitotic rate $<10$ per 50 high-power fields with clear resection margins and without distant metastases and radical surgery offers probably no benefit in survival in these patients $[38,39]$. In recent years, several large series reported good results with excellent oncological outcome and low complication profile after rendezvous resection of GISTs and other gastric submucosal tumors [2-5, 19]. Our experiences confirm these findings.

When compared with conventional gastric surgery, laparoscopic-endoscopic rendezvous resection of upper gastrointestinal tumors has several clinical advantages, including less pain, less inflammatory response, faster recovery of gut function, shorter hospital stay, less hospital charges and better quality of life [3-5]. What appears to be only in the large series comparing open and laparoscopic gastric resection of stromal tumors, a significant shorter hospital stay was reported for the laparoscopic group [2].

In summary, a variety of minimally invasive surgical techniques may be successfully employed in the treatment of a wide array of gastric pathologies. Although the outcome data of laparoscopic-endoscopic rendezvous resection are in its infancy, the early results are promising. 


\section{References}

1 Jacobaeus HC: Über die Möglichkeit die Zystoskopie bei Untersuchungen seröser Höhlungen anzuwenden. Munch Med Wochenschr 1910;57:2090-2092.

-2 Matthews BD, Walsh RM, Kercher KW, Sing RF, Pratt BL, Answini GA, Heniford BT: Laparoscopic vs. open resection of gastric stromal tumors. Surg Endosc 2002;16:803-807.

-3 Bouillot JL, Bresler L, Fagniez PL, Samama G, Champault G, Parent Y: Laparoscopic resection of benign submucosal stomach tumors. A report of 65 cases. Gastroenterol Clin Biol 2003;27:272-276.

4 Pross M, Wolff S, Schubert D, Meyer L, Lippert $\mathrm{H}$ : Combined minimal-invasive procedures for resection of benign gastric wall tumors. Zentralbl Chir 2003;128:191-194.

5 Ludwig K, Weiner R, Bernhardt J: Minimally invasive resections of gastric tumors. Chirurg 2003;74:632-637.

-6 Avital S, Brasesco O, Szomstein S, Liberman M, Rosenthal R: Technical considerations in laparoscopic resection of gastric neoplasms. Surg Endosc 2003;17:763-765.

7 Adachi Y, Shiraishi N, Kitano S: Modern treatment of early gastric cancer: review of the Japanese experience. Dig Surg 2002;19:333-339.

8 Aogi K, Hirai T, Mukaida H, Toge T, Haruma K, Kajiyama G: Laparoscopic resection of submucosal gastric tumors. Surg Today 1999;29: 102-106.

-9 Gurbuz AT, Peetz ME: Resection of a gastric leiomyoma using combined laparoscopic and gastroscopic approach. Surg Endosc 1997;11: 285-286.

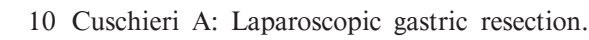
Surg Clin North Am 2000;80:1269-1284.

$\checkmark 11$ Matsumoto Y, Yanai H, Tokiyama H, Nishiaki M, Higaki S, Okita K: Endoscopic ultrasonography for diagnosis of submucosal invasion in early gastric cancer. J Gastroenterol 2000; 35:326-331.

12 Willis S, Truong S, Gribnitz S, Fass J, Schumpelick V: Endoscopic ultrasonography in the preoperative staging of gastric cancer: accuracy and impact on surgical therapy. Surg Endosc 2000;14:951-954.

13 Gress F, Schmitt C, Savides T, Faigel DO, Catalano M, Wassef W, Roubein L, Nickl, N, Ciaccia D, Bhutani M, Hoffman B, Affronti J: Interobserver agreement for EUS in the evaluation and diagnosis of submucosal masses. Gastrointest Endosc 2001;53:71-76.

14 Wakelin SJ, Deans C, Crofts TJ, Allan PL, Plevris JN, Paterson-Brown SA: Comparison of computerised tomography, laparoscopic ultrasound and endoscopic ultrasound in the preoperative staging of oesophago-gastric carcinoma. Eur J Radiol 2002;41:161-167.
15 Adrales GL, Gandsas A, Mastrangelo MJ Jr, Schwartz R: An introduction to laparoscopic gastric resection. Curr Surg 2003;60:385389.

16 Ohashi S: Laparoscopic intraluminal (intragastric) surgery for early gastric cancer. A new concept in laparoscopic surgery. Surg Endosc 1995;9:169-171.

17 Tagaya N, Kita J, Kogure H, Kubota K: Laparoscopic intragastric resection of gastric leiomyoma using needlescopic instruments. Case report. Surg Endosc 2001;15:414.

18 Stellato TA: Flexible endoscopy as an adjunct to laparoscopic surgery. Surg Clin North Am 1996;76:595-602.

19 Ludwig K, Wilhelm L, Scharlau U, Amtsberg G, Bernhardt J: Laparoscopic-endoscopic rendezvous resection of gastric tumors. Surg Endosc 2002;16:1561-1565.

20 Palazzo L, Landi B, Cellier C, Cuillerier E, Roseau G, Barbier JP: Endosonographic features predictive of benign and malignant gastrointestinal stromal cell tumours. Gut 2000;46: 88-92.

- 21 Siewert JR, Bottcher K, Stein HJ, Roder JD: Relevant prognostic factors in gastric cancer: ten-year results of the German Gastric Cancer Study. Ann Surg 1998;228:449-461.

- 22 Nakamura K, Ueyama T, Yao T, Xuan ZX, Ambe K, Adachi Y, Yakeishi Y, Matsukuma A, Enjoji M: Pathology and prognosis of gastric carcinoma. Findings in 10,000 patients who underwent primary gastrectomy. Cancer 1992; 70:1030-1037.

23 Kim JP, Hur YS, Yang HK: Lymph node metastasis as a significant prognostic factor in early gastric cancer: analysis of 1,136 early gastric cancers. Ann Surg Oncol 1995;2:308-313.

24 Kunisaki C, Shimada H, Takahashi M, Ookubo K, Moriwaki Y, Akiyama H, Nomura M: Prognostic factors in early gastric cancer. Hepatogastroenterology 2001;48:294-298.

25 Nakamura K, Morisaki T, Sugitani A, Ogawa T, Uchiyama A, Kinukawa N, Tanaka M: An early gastric carcinoma treatment strategy based on analysis of lymph node metastasis. Cancer 1999;85:1500-1505.

26 Gotoda T, Yanagisawa A, Sasako M, Ono H, Nakanishi Y, Shimoda T, Kato Y: Incidence of lymph node metastasis from early gastric cancer: Estimation with a large number of cases at two large centers. Gastric Cancer 2000; 3:219-225.

27 Yamada H, Nihei Z, Yamashita T, Shirota Y, Ichikawa W, Sugihara K: Is lymphadenectomy needed for all submucosal gastric cancers? Eur J Surg 2001;167:199-203.

28 Yasuda K, Shiraishi N, Suematsu T, Yamaguchi K, Adachi Y, Kitano S: Rate of detection of lymph node metastasis is correlated with the depth of submucosal invasion in early stage gastric carcinoma. Cancer 1999;85:21192123.
29 Tanabe S, Koizumi W, Mitomi H, Nakai H, Murakami S, Nagaba S, Kida M, Oida M, Saigenji K: Clinical outcome of endoscopic aspiration mucosectomy for early stage gastric cancer. Gastrointest Endosc 2002;56:708713.

30 Miyamoto S, Muto M, Hamamoto Y, Boku N, Ohtsu A, Baba S, Yoshida M, Ohkuwa M, Hosokawa K, Tajiri H, Yoshida S: A new technique for endoscopic mucosal resection with an insulated-tip electrosurgical knife improves the completeness of resection of intramucosal gastric neoplasms. Gastrointest Endosc 2002; 55:576-581.

- 31 Yamamoto H, Kawata H, Sunada K, Satoh K, Kaneko Y, Ido K, Sugano K: Success rate of curative endoscopic mucosal resection with circumferential mucosal incision assisted by submucosal injection of sodium hyaluronate. Gastrointest Endosc 2002;56:507-512.

32 Ono H, Kondo H, Gotoda T, Shirao K, Yamaguchi H, Saito D, Hosokawa K, Shimoda T, Yoshida S: Endoscopic mucosal resection for treatment of early gastric cancer. Gut 2001;48: 225-229.

33 Hiki Y, Sakuramoto S, Katada N, Shimao H: Combined laparoscopic-endoscopic procedure in stomach carcinoma. Chirurg 2000;71:11931201.

-34 Kobayashi T, Kazui T, Kimura T: Surgical local resection for early gastric cancer. Surg Laparosc Endosc Percutan Tech 2003;13:299_ 303.

35 Seto Y, Yamaguchi H, Shimoyama S, Shimizu N, Aoki F, Kaminishi M: Results of local resection with regional lymphadenectomy for early gastric cancer. Am J Surg 2001;182:498-501.

36 Ludwig K, Bernhardt J, Weiner R: Rendezvousverfahren am Magen. Chir Gastroenterol 2004;19:106-115.

37 Nakahara K, Tsuruta O, Tateishi H, Arima N, Takeda J, Toyonaga A, Sata M: Extended indication criteria for endoscopic mucosal resection of early gastric cancer with special reference to lymph node metastasis - examination by multivariate analysis. Kurume Med J 2004; 51:9-14.

38 Fletcher CD, Berman JJ, Corless C, Gorstein F, Lasota J, Longley BJ, Miettinen M, O'Leary TJ, Remotti H, Rubin BP, Shmookler B, Sobin LH, Weiss SW: Diagnosis of gastrointestinal stromal tumors: a consensus approach. Hum Pathol 2002;33:459-465.

39 DeMatteo RP, Lewis JJ, Leung D, Mudan SS Woodruff JM, Brennan MF: Two hundred gastrointestinal stromal tumors: recurrence patterns and prognostic factors for survival. Ann Surg 2000;231:51-58. 